\title{
Exploiting optical near fields for phase change memories
}

\author{
P. Leiprecht, ${ }^{1,2}$ P. Kühler, ${ }^{2}$ M. Longo, ${ }^{3}$ P. Leiderer, ${ }^{2}$ C. N. Afonso, ${ }^{1}$ and J. Siegel ${ }^{1, a)}$ \\ ${ }^{1}$ Laser Processing Group, Instituto de Optica, CSIC, Serrano 121, 28006 Madrid, Spain \\ ${ }^{2}$ Faculty of Physics, University of Konstanz, Universitätsstraße 10, 78457 Konstanz, Germany \\ ${ }^{3}$ Laboratorio MDM, IMM-CNR, Via C. Olivetti 2, I-20041 Agrate Brianza, MB, Italy
}

(Received 17 November 2010; accepted 11 December 2010; published online 4 January 2011)

\begin{abstract}
We apply a recently developed technique based on optical near fields to achieve reversible phase switching in $\mathrm{Ge}_{2} \mathrm{Sb}_{2} \mathrm{Te}_{5}$ films. By placing dielectric microspheres at the film surface and exposing them to pulsed laser light, a complex intensity distribution due to the optical near field can be created at the film surface. We demonstrate writing and erasing operations of patterns through phase switching. Spheres can be removed after an operation by optical near fields without ablation. Data erasure is achieved with and without near fields. The erasure method used can be determined from the result and erased information can be retrieved although being inverted. Three distinct material states are identified within patterns, showing clear contrast and sharp borders between them, thus opening the possibility of three-level data storage. Our results suggest that optical near fields are a promising candidate for developing strategies in data storage, encryption, and multiplexing. (C) 2011 American Institute of Physics. [doi:10.1063/1.3533395]
\end{abstract}

Phase change data storage is based on switching of chalcogenide films between the amorphous and crystalline phase by means of controlled heating and cooling, typically using ns laser or electrical pulses. ${ }^{1,2}$ The two phases have very different optical and electrical properties, which can be read out optically or electrically. Phase change optical disks have appeared already in 1990 as a commercial product and electrical phase change memories (PCM) are under development. $\mathrm{Ge}_{2} \mathrm{Sb}_{2} \mathrm{Te}_{5}$ (GST) is one of the material compositions of choice for optical and electrical PCM.

Optical near fields (ONFs) open the possibility of breaking the diffraction limit of light. ${ }^{3}$ Because of this exceptional feature, they have become a field of intense study with two major aims. First, to achieve a better comprehension of the complex structure of ONFs that is highly sensitive to a specific situation. Second, to identify applications for which farfield techniques fail to deliver the performance required. As for the study of ONFs, methods such as near field ablation, ${ }^{4}$ scanning near field optical microscopy, ${ }^{5}$ and modeling ${ }^{6}$ have been applied. Very recently, near field phase change ${ }^{7,8}$ has been introduced, providing superior sensitivity, contrast, and precision together with linear response. As for applications, ONFs have already been established in material processing, ${ }^{9,10}$ microscopy, ${ }^{3}$ and biosensing, ${ }^{11}$ showing an astonishing performance with subdiffraction resolution.

In the field of phase change memories, ONFs have been implemented so far in form of super resolution near field structure (super-RENS) optical storage concepts, as reported in the pioneering work of Tominaga and co-workers. ${ }^{12}$ In that specific application, ONFs are created upon illumination of an individual subwavelength aperture within a patterned optical disk. The resulting intensity enhancement behind the aperture is exploited to write binary information in a Pt oxide layer, with the phase change layer acting as a transient lens. ${ }^{13}$

In the present study, we demonstrate the enormous potential of the 3D nature of ONFs for information storage rather than focusing on the capability of ONFs to scale down

${ }^{a)}$ Electronic mail: j.siegel@io.cfmac.csic.es. the bit size, as done in super-RENS. In particular, we demonstrate writing and erasing operations of highly complex patterns employing ONFs generated in the vicinity of dielectric microspheres sitting on a phase change layer.

Samples consisted of 40-nm-thick fcc crystalline GST films sputter-deposited onto a 10 -nm-thick $\mathrm{SiO}_{2}$ layer covering a $\mathrm{Si}$ wafer. Spherical silica spheres with different diameters $(\varnothing=4.66 \mu \mathrm{m}$ and $990 \mathrm{~nm})$ were deposited on these films under conditions that ensure particle isolation. The laser used for performing experiments was an ArF excimer laser (wavelength $\lambda=193 \mathrm{~nm}, 20 \mathrm{~ns}$ pulse duration). An ad hoc designed configuration ${ }^{8}$ allowed producing a top-hat intensity distribution of the beam incident on the sample at a user-selected angle $\theta$ and fluence $F$. After irradiation, the patterns were imaged using optical microscopy (OM) and field emission scanning electron microscopy (SEM).

Figure 1(a) shows the pattern written by the ONF of a $4.6 \mu \mathrm{m}$ sphere after exposure to a single laser pulse. The imprint consists of an elliptical periodic pattern, in which the dark rings correspond to laser-amorphized regions (high local field intensities) and the bright rings to nonamorphized or little-amorphized regions (low intensities). The ellipticity is caused by the angled incidence (from the left). The period along the forward direction in the far field has been measured to be $p_{f w, \exp } \approx 1050 \mathrm{~nm}$, in agreement with the expected behavior $p_{f w, \text { calc }}=\lambda /(1-\sin \theta)=1010 \mathrm{~nm}$. While angle dependence of near field patterns of microspheres on GST films upon excimer laser irradiation has previously been studied, ${ }^{6}$ the use of amorphous films (instead of crystalline as in our case) prevented the authors from achieving phase switching.

Since the sphere remains after laser irradiation [cf. Fig. 1(a)], it obstructs the inspection of the rings in the backward direction. The sphere (marked by a dashed circle) is surrounded by circular concentric rings and shadow regions, which are caused by an optical effect of the defocused sphere and should be ignored. The dark spot to the right of the sphere is the ablation crater caused by the strong lens effect yielding a focus with a strong field enhancement. 


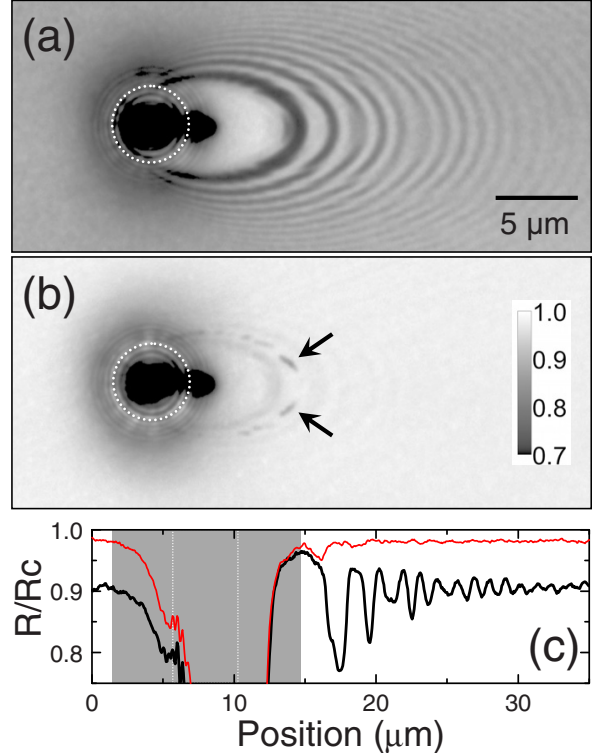

FIG. 1. (Color online) Optical micrographs of (a) writing and (b) erasing operations of patterns, exploiting optical near fields surrounding a silica sphere $\left(\varnothing=4.66 \mu \mathrm{m}\right.$, highlighted by a dotted circle) on a $\mathrm{Ge}_{2} \mathrm{Sb}_{2} \mathrm{Te}_{5}$ film. (a) Single laser pulse $\left(F=75 \mathrm{~mJ} / \mathrm{cm}^{2}\right)$ at an angle of incidence of $54^{\circ}$. (b) Exposure of (a) to a second pulse $\left(F=33 \mathrm{~mJ} / \mathrm{cm}^{2}\right)$ at $54^{\circ}$. The grayscale displays relative reflectivity changes with respect to the reflectivity of the crystalline film $(R=1)$. The arrows indicate the regions of incomplete erasure. (c) Horizontal normalized reflectivity profiles through the image center of (a) (thick line) and (b) (thin line)

The pattern written in Fig. 1(a) has been erased by exposing the very same region, with the sphere present, to a second laser pulse at about half the fluence of the writing pulse. This fluence ratio is typical for writing/erasing operations in GST, triggering melting-induced amorphization/ heating-induced crystallization, respectively. Figure 1(b) displays the result, showing an overall recovery in reflectivity of the region surrounding the sphere together with an erasure of the pattern, consistent with recrystallization. Apart from the sphere and the ablation crater, both still present, a few regions close to the sphere still exhibit residual amorphous features. We tentatively attribute the existence of these residual features to a slight change in the ONF distribution. The local ONF is expected to differ for substrate regions in different phases and thus optical properties.

In order to evaluate if the erasure operation has been sufficiently complete in terms of distinguishing both states (written-erased) nonambiguously, we have plotted the reflectivity profiles [Fig. 1(c)]. The profile after the writing operation shows an average reflectivity decrease down to $R_{\text {write-av }} \approx 0.90$, as can be seen at $x=0$ and $35 \mu \mathrm{m}$, indicating partial amorphization. The superimposed near field generated modulation has a depth of $\Delta R_{\text {write-ONF }}=0.96-0.77=0.19$ at $x=15-17 \mu \mathrm{m}$, with 0.77 corresponding to almost complete amorphization. In comparison, the profile after erasure shows that the reflectivity (and thus crystallinity) recovers up to $R_{\text {erase-av }}=0.98$ (except the shadow and ablation region), well above $R_{\text {write-av }}$ and significantly above any modulation peak induced by the writing operation. This demonstrates the viability of near field writing and near field erasure, making use of the same dielectric sphere.

A drawback for applications could be the remaining sphere after a writing operation. In particular, if the purpose is to write a pattern but to avoid near field erasure, it might

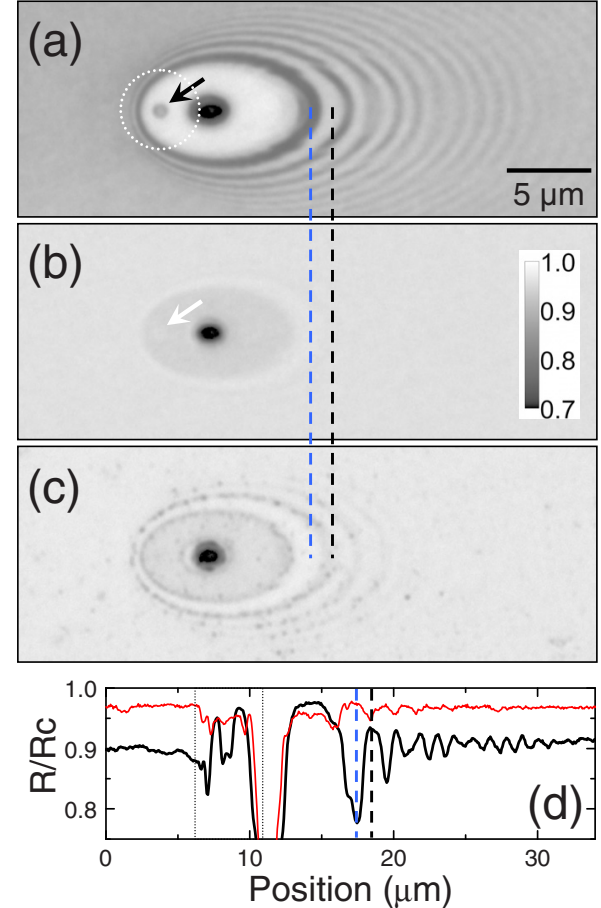

FIG. 2. (Color online) (a) Optical micrograph of a pattern recorded under the same conditions as in Fig. 1(a), after exposure to a second pulse at normal incidence and low fluence $\left(F=2.7 \mathrm{~mJ} / \mathrm{cm}^{2}\right)$, to remove the sphere. The original position of the sphere is marked by a dotted circle and the arrow points at the so-produced amorphous spot. (b) Optical micrograph of the same region as in (a) after exposure to three pulses at normal incidence and $F=12 \mathrm{~mJ} / \mathrm{cm}^{2}$. The arrow points at the so-produced recrystallized spot. (c) Optical micrograph of the same region as in (b) after exposure to a further single pulse at normal incidence $\left(F=33 \mathrm{~mJ} / \mathrm{cm}^{2}\right)$, causing pattern retrieval. (c) Horizontal reflectivity profiles through the image center of (a) (thick line) and (c) (thin line). The vertical dashed lines in (a)-(d) are a guide to the eye to show that the written and retrieved patterns are inverted

be useful to remove the sphere. While there are several mechanical methods ${ }^{14}$ to remove particles, we have applied laser cleaning ${ }^{15}$ since it allows in-situ removal. Changing the angle of incidence to $\theta=0^{\circ}$ and lowering the laser fluence to $F=2.7 \mathrm{~mJ} / \mathrm{cm}^{2}$, the sphere is removed, leaving behind a small amorphous spot at the contact point with the film [Fig. 2(a)]. In this way, the ONF pattern in the backward direction can be accessed, whose period in the far field should scale accordingly as $p_{b w, \text { calc }}=\lambda /(1+\sin \theta)=107 \mathrm{~nm}$. Obviously, this cannot be resolved by $\mathrm{OM}$ and only two amorphous rings can be distinguished, close to the contact point where the far-field approximation is not valid.

With the sphere removed, we investigated if the pattern can be erased in the absence of a sphere, i.e., in a conventional way without ONFs. For that purpose we have determined the optimum fluence for erasing the pattern by exposure to a single laser pulse at $\theta=0^{\circ}$, yielding $F_{\text {conv }}$ $=12 \mathrm{~mJ} / \mathrm{cm}^{2}$. Full pattern erasure required at least three consecutive exposures at this fluence, the result of which is shown in Fig. 2(b). In contrast to near field erasure [cf. Fig. 1(b)], conventional erasure modifies the "shadow" region, originally shielded by the sphere, causing partial amorphization. The result of near field erasure can thus be clearly distinguished from conventional erasure, which is a useful property for data security. A further detail can be found upon inspection of Fig. 2(b). The small amorphous spot produced upon removing the sphere switches back to bright [cf. arrows 


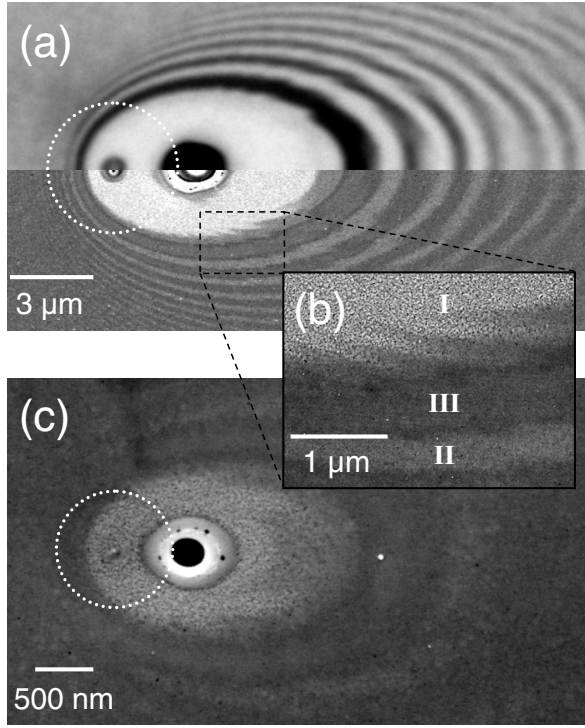

FIG. 3. (a) Superposition of an optical micrograph (upper part) and SEM image (lower part) of a pattern written under the same conditions as in Fig. 2(a). (b) High resolution SEM image of a small region of (a), showing three different, steplike regimes (I crystalline, II partial amorphous, and III amorphous). (c) SEM image of a pattern written using a smaller sphere $(\varnothing$ $=990 \mathrm{~nm}$ ), showing three regimes and a tiny modification at the original contact point of the sphere with the film.

in Figs. 2(a) and 2(b)], thus confirming that phase switching is possible also at the contact point.

As a last step of conventional erasure, we have investigated the possibility of data retrieval, i.e., of recovering a written pattern after erasure. This is possible, as demonstrated in Fig. 2(c) showing the same region after exposure to a single laser pulse of intermediate fluence $F_{\text {ret }}$ $=36 \mathrm{~mJ} / \mathrm{cm}^{2}$. It seems that the pattern can be recovered, although its contrast is lower than in Fig. 2(a). However, close inspection reveals that the retrieved pattern is inverted as can be seen by the vertical lines in Figs. 2(a)-2(c) and in the reflectivity profiles in Fig. 2(d).

In order to overcome the resolution limit of OM, we have employed field emission SEM in backscattering configuration to image patterns with high spatial resolution. ${ }^{8}$ Figure 3(a) shows a direct comparison of images recorded with OM and SEM of a pattern similar to the one shown in Fig. 2(a). The equivalent response (dark-amorphous, brightcrystalline) in both imaging modes can be appreciated. The superior resolution using SEM reveals that there are three, nicely delineated regimes, which are associated with crystalline (bright, regime I), fully amorphized (dark, regime III), and partially amorphized (gray, regime II) regions. The existence of three regimes instead of two opens the possibility of multilevel storage. ${ }^{16}$ The complexity of the imprinted ONF pattern can be appreciated also in the zoomed region [Fig. $3(\mathrm{~b})$ ], revealing the polycrystalline nature of the crystalline region and the smoothing of the surface morphology upon partial and full amorphization. The borders between the three regimes are somewhat sharper for the transition from regimes II to III than for the transitions I to II. A factor limiting the sharpness of features is the laser pulse duration $(20 \mathrm{~ns})$, yielding a thermal diffusion length of $\approx 100 \mathrm{~nm}^{8}$

We have also generated an imprint of the ONF of a smaller sphere [990 nm, c.f. Fig. 3(c)]. Despite the blurring of features due to thermal diffusion, characteristic features of the pattern and the three regimes can be distinguished. What is remarkable is the small spot produced at the original contact point between sphere and film which measures approximately $50 \mathrm{~nm}$ in diameter. We attribute the existence of such a small feature to the strong threshold behavior of the material, ${ }^{17}$ partially compensating for the limitations imposed by lateral heat flow. The influence of heat flow can be reduced by using femtosecond laser pulses and features with $55 \mathrm{~nm}$ in diameter have been achieved using microlens arrays, despite the longer wavelength used $(800 \mathrm{~nm}){ }^{18}$

In summary, we have demonstrated the possibility of exploiting optical near fields of microspheres for writing and erasing of patterns in phase change materials. Data erasure of patterns can be performed with or without near fields and the erasure method used can be determined from the erased region by direct comparison; an important feature for data security. Moreover, erased patterns can be retrieved in a simple way as inverted patterns. The complexity and twodimensional nature of the written patterns in combination with the strong dependence on the experimental writing conditions should open possibilities for developing strategies in data storage, encryption, and multiplexing.

The authors thank E. Varesi, A. Pirovano, and R. Bez from Numonyx, Agrate Brianza, Italy for supplying the GST films. The research was performed within a Joint Project between CSIC and Konstanz University funded by the Spanish Government and the DAAD, as well as within a Bilateral Agreement between CSIC and CNR. We also acknowledge partial funding from Spanish National Research Projects (Grants No. TEC2008-01183 and No. TEC2008-03379-E), and by Deutsche Forschungsgemeinschaft (SFB 767 and SPP 1327).

${ }^{1}$ J. Feinleib, J. deNeufville, S. C. Moss, and S. R. Ovshinsky, Appl. Phys. Lett. 18, 254 (1971).

${ }^{2}$ S. R. Ovshinsky, Phys. Rev. Lett. 21, 1450 (1968).

${ }^{3}$ N. I. Zheludev, Nature Mater. 7, 420 (2008)

${ }^{4}$ P. Leiderer, C. Bartels, J. König-Birk, M. Mosbacher, and J. Boneberg, Appl. Phys. Lett. 85, 5370 (2004).

${ }^{5}$ R. Hillenbrand, F. Keilmann, P. Hanarp, P. S. Sutherland, and J. Aizpuru, Appl. Phys. Lett. 83, 368 (2003).

${ }^{6}$ Z. B. Wang, M. H. Hong, B. S. Luk'yanchuk, Y. Lin, Q. F. Wang, and T. C. Chong, J. Appl. Phys. 96, 6845 (2004).

${ }^{7}$ P. Kühler, F. J. García de Abajo, J. Solis, M. Mosbacher, P. Leiderer, C. N. Afonso, and J. Siegel, Small 5, 1825 (2009).

${ }^{8}$ J. Siegel, D. Puerto, J. Solis, F. J. García de Abajo, C. N. Afonso, M. Longo, C. Wiemer, M. Fanciulli, P. Kühler, M. Mosbacher, and P. Leiderer, Appl. Phys. Lett. 96, 193108 (2010).

${ }^{9}$ S. M. Huang, M. H. Hong, B. Luk'yanchuk, and T. C. Chong, Appl. Phys. A: Mater. Sci. Process. 77, 293 (2003).

${ }^{10}$ E. McLeod and C. B. Arnold, Nat. Nanotechnol. 3, 413 (2008).

${ }^{11}$ S. J. Lee, Z. Guan, H. Xu, and M. Moskovits, J. Phys. Chem. C 111, 17985 (2007).

${ }^{12}$ J. Tominaga, T. Nakano, and N. Atoda, Appl. Phys. Lett. 73, 2078 (1998)

${ }^{13} \mathrm{~J}$. Tominaga, in Phase Change Materials: Science and Applications, edited by S. Raoux and M. Wuttig (Springer, New York, 2009), pp. 285-298.

${ }^{14} \mathrm{~J}$. Bardina, in Particles on Surfaces, edited by K. L. Mittal (Plenum, New York, 1988), pp. 329-337.

${ }^{15}$ K. Imen, J. Lee, and S. D. Allen, Appl. Phys. Lett. 58, 203 (1991).

${ }^{16}$ B.-S. Lee and S. G. Bishop, in Phase Change Materials: Science and Applications, edited by S. Raoux and M. Wuttig (Springer, New York, 2009), pp. 187-188.

${ }^{17}$ J. Siegel, W. Gawelda, D. Puerto, C. Dorronsoro, J. Solis, C. Afonso, J. C. G. de Sande, R. Bez, A. Pirovano, and C. Wiemer, J. Appl. Phys. 103, 023516 (2008).

${ }^{18}$ Y. Lin, M. H. Hong, C. S. Lim, G. X. Chen, L. S. Tan, Z. B. Wang, L. P. Shi, and T. C. Chong, Appl. Phys. Lett. 89, 041108 (2006). 